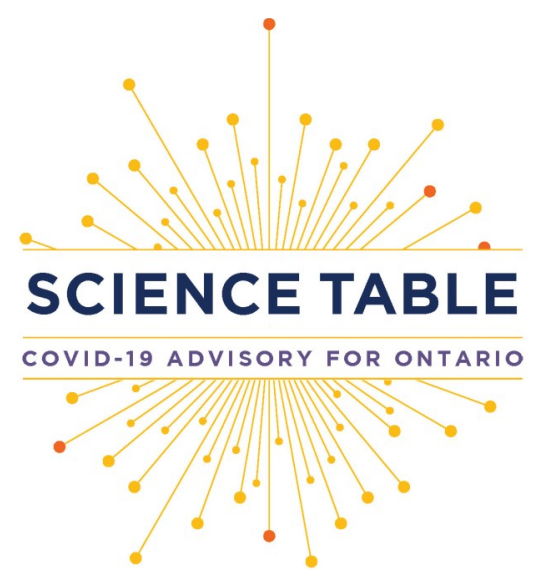

Version 1.1

Published: March 8, 2021

Updated on March 8, 2021. Version 1.0 is available under Additional Resources at https://doi.org/10.47326/ocsat.2021.02.13.1.0.

Citation: Brown KA, Stall NM, Vanniyasingam T, et al. Early impact of Ontario's COVID-19 vaccine rollout on long-term care home residents and health care workers. Science Briefs of the Ontario COVID-19 Science Advisory Table. 2021;2(13). https:// doi.org/10.47326/ocsat.2021.02.13.1.0

Author Affiliations: The affiliations of the members of the Ontario COVID-19 Science Advisory Table can be found at https:// covid19-sciencetable.ca/.

Declarations of Interest: The declarations of interest of the members of the Ontario COVID-19 Science Advisory Table can be found at https://covid19-sciencetable.ca/.

About Us: The Ontario COVID-19 Science Advisory Table is a group of scientific experts and health system leaders who evaluate and report on emerging evidence relevant to the COVID-19 pandemic, to inform Ontario's response. Our mandate is to provide weekly summaries of relevant scientific evidence for the COVID-19 Health Coordination Table of the Province of Ontario, integrating information from existing scientific tables, Ontario's universities and agencies, and the best global evidence. The Science Table summarizes its findings for the Health Coordination Table and the public in Science Briefs.

The Congregate Care Setting Working Group is a group of internationally recognized researchers with expertise in older people living in congregate care settings. The Working Group evaluates emerging scientific evidence related to congregate care settings to inform Ontario's response to the COVID19 pandemic. The Working Group reports its findings to the public and the Science Table. Its findings are also summarized in Science Briefs.

Correspondence to: Secretariat of the Ontario COVID-19 Science Advisory Table (info@covid19-sciencetable.ca)

\title{
Early Impact of Ontario's COVID-19 Vaccine Rollout on Long-Term Care Home Residents and Health Care Workers
}

Kevin A. Brown, Nathan M. Stall, Thuva Vanniyasingam, Sarah A. Buchan, Nick Daneman, Michael P. Hillmer, Jessica Hopkins, Jennie Johnstone, Antonina Maltsev, Allison McGeer, Beate Sander, Rachel D. Savage, Tania Watts, Peter Jüni, Paula A. Rochon on behalf of the Congregate Care Setting Working Group and the Ontario COVID-19 Science Advisory Table

\section{Key Message}

The rollout of COVID-19 vaccines to Ontario's long-term care (LTC) homes has substantially reduced SARS-CoV-2 infections, COVID-19 hospitalizations, and deaths among LTC residents and health care workers.

Completing and maximizing the uptake of the full COVID-19 vaccine series according to recommended schedules will maximize the safety and well-being of Ontario's LTC residents and staff.

\section{Summary}

\section{Background}

While only accounting for $0.5 \%$ of Ontario's population, long-term care (LTC) residents across the province have had disproportionately high rates of SARS-COV-2 infections and COVID-19 deaths.

Ontario's COVID-19 vaccine rollout began in mid-December 2020, with LTC residents and staff identified as Phase 1 priority populations for vaccination.

\section{Question}

What was the early impact of the COVID-19 vaccine on SARS-CoV-2 cases, COVID-19 hospitalizations and deaths among Ontario's LTC residents and health care workers (HCWs)?

\section{Findings}

LTC home staff were the first to receive the vaccine in clinics starting on December 14, 2020. Most LTC home residents started receiving first doses of the COVID-19 vaccine after December 23, 2020. All LTC residents in Ontario were offered at least the first dose of a COVID-19 vaccine by February 13, 2021.

As of February 23, 2021, more than 64,000 Ontario LTC residents (92\%) received at least one dose of a COVID-19 vaccine, with over 46,500 of residents having received both doses. Over 55,000 Ontario LTC staff (55\%) also received at least one dose of a COVID-19 vaccine, with more than 44,600 having received both doses.

As of February 23, 2021, COVID-19 vaccination in LTC homes prevented an estimated 2,079 SARS-CoV-2 infections, 249 COVID-19 hospitalizations, and 615 COVID-19 deaths in residents, and an estimated 330 SARS-CoV-2 infections, and 8 COVID-19 hospitalizations and 1 COVID-19 death in HCWs. 
Copyright: 2021 Ontario COVID-19 Science Advisory Table. This is an open access document distributed under the terms of the Creative Commons Attribution License, which permits unrestricted use, distribution, and reproduction in any medium, provided that the original work is properly cited.

The views and findings expressed in this Science Brief are those of the authors and do not necessarily reflect the views of all of the members of the Ontario COVID-19 Science Advisory Table, its Working Groups, and its partners.
Eight weeks after the start of vaccination, the estimated relative reduction in SARSCoV-2 incidence was $89 \%$ in LTC residents and $79 \%$ in LTC HCWs. The estimated relative reduction in COVID-19 mortality in LTC residents was $96 \%$ after 8 weeks.

\section{Interpretation}

The rollout of COVID-19 vaccines in Ontario's LTC homes substantially reduced SARS-CoV-2 infections, COVID-19 hospitalizations and deaths among LTC residents and HCWs. Completing and maximizing the uptake of the full COVID-19 vaccine series according to recommended schedules will maximize the safety and well-being of Ontario's LTC residents and staff.

\section{Background}

In Ontario there are 626 LTC homes with 69,799 residents occupying 77,257 longstay beds as of January 2021. ${ }^{1,2}$ Fifty-five percent of Ontario's LTC residents are 85 years of age or older ${ }^{3}$ and $70 \%$ are women. ${ }^{4}$ Ontario's LTC homes employ approximately 100,000 clinical and non-clinical staff, who are involved in clinical work, caregiving, administration, housekeeping, food preparation, facilities management, maintenance, and recreation. ${ }^{5}$ The majority of personal care for LTC residents is provided by approximately 50,000 personal support workers (PSWs), who represent the largest group of employees in the LTC sector, followed by approximately 25,000 registered practical nurses, registered nurses, and nurse practitioners. Nearly 90\% of Ontario's PSWs are women, half are between the ages of 35 and 54 years, and more than $40 \%$ are visible minorities. ${ }^{5}$

While accounting for only $0.5 \%$ of Ontario's population of 15 million, LTC residents across the province have had disproportionately high rates of SARS-COV-2 infections and COVID-19 deaths. As of February 23, 2021, there has been a total of 14,958 cumulative SARS-CoV-2 infections and 3,858 cumulative COVID-19 deaths among LTC residents (55\% of Ontario's 6,940 COVID-19 deaths).

Ontario's COVID-19 vaccine rollout began in mid-December 2020, with LTC residents and staff identified as Phase 1 priority populations for vaccination. ${ }^{6}$ The PfizerBioNTech vaccine was the first COVID-19 vaccine approved by Health Canada on December 9, 2020, and also the first vaccine received in the Province of Ontario. Due to vaccine stability concerns and regulations about the storage, handling, and transportation of the Pfizer-BioNTech vaccine, Ontario LTC staff were the first to receive the vaccine in hospital-based clinics starting on December 14, 2020. Most LTC home residents received the Moderna vaccine, with vaccination starting after Health Canada's approval on December 23, 2020.

Ontario's COVID-19 vaccine rollout began alongside a range of public health measures implemented by the Province of Ontario to reduce community transmission of SARS-CoV-2. This included a province-wide shutdown beginning on December 26, 2020, followed by a province-wide stay-at-home order commencing on January $14,2021 .^{7}$ On February 22,2021 , all Ontario regions had schools return to in-class learning, and all regions except for Toronto, Peel, and North Bay-Parry Sound lifted some of their public health restrictions, in accordance with a return to Ontario's COVID-19 response framework. ${ }^{8}$

\section{Question}

What was the early impact of the COVID-19 vaccine on SARS-CoV-2 cases, COVID-19 hospitalizations and deaths among Ontario's LTC residents and health care workers? 


\section{Findings}

Prioritization of Ontario LTC homes for COVID-19 Vaccination and Speed of the Vaccine Rollout

In the pre-vaccination period between November 1 and December 13, 2020, there were 2,433 reported SARS-CoV-2 infections among LTC residents, and 991 among LTC HCWs. The COVID-19 vaccine rollout started in Ontario on December 14, 2020, with LTC homes in the public health units (PHUs) of Toronto, Peel, York, and Windsor-Essex prioritized for initial allocation of vaccine due to those regions having the highest SARS-CoV-2 infection rates.

The Province set January 21, 2021, as the target date for the provision of first doses of COVID-19 vaccines to LTC residents residing in those high priority regions, and February 10, 2021, as the target date for the provision of first doses to all remaining LTC residents across Ontario. ${ }^{9}$ All LTC residents in high priority regions were offered the first dose of a COVID-19 vaccine by January 19, 2021. All remaining LTC residents were offered the first dose of a COVID-19 vaccine by February 13, 2021.

\section{COVID-19 Vaccination of Ontario LTC home Residents and Staff}

As of February 23, 2021, more than 64,000 Ontario LTC residents received at least one dose of a COVID-19 vaccine (92\%), with over 46,500 of residents having received both doses. Over 55,000 LTC staff have received at least one dose of a COVID-19 vaccine (55\%), with more than 44,600 having received both doses.

As of March 5, 2021, over 66,000 LTC residents received at least one dose of a COVID-19 vaccine (95\%) with 55,600 having received both doses. Over 67,000 LTC staff have received at least one dose of a COVID-19 vaccine (68\%), with over 47,500 having received both doses.

\section{Early Impact of Ontario's COVID-19 Vaccine Rollout on LTC Home Residents and} HCWs

The cumulative number SARS-CoV-2 infections reported between November 1, 2020, and February 23, 2021, was 8,007 among LTC residents and 3,625 among LTC HCWs. LTC residents with SARS-CoV-2 infections were on average 85 years of age, and $67 \%$ were women. LTC HCWs with SARS-CoV-2 infections were on average 43 years of age and $88 \%$ were women.

Figure 1 (A) shows the daily number of SARS-CoV-2 infections observed in LTC residents during this period as compared with community dwelling older adults aged 70 years or above in Ontario who had not yet access to COVID-19 vaccine. ${ }^{10}$ Figure 1 (B) presents the daily number of SARS-CoV-2 infections found in observed in LTC $\mathrm{HCW}$ as compared with working age adults aged 18-64 years.

A decline in SARS-CoV-2 infections was demonstrated for both LTC home residents and community-dwelling older adults, approximately 10 days following the implementation of the Ontario government's province-wide shutdown on December 26, 2020. However, the reduction in SARS-CoV-2 infections in LTC residents and $\mathrm{HCWs}$, considerably exceeded the reductions seen in the control populations. 
Residents

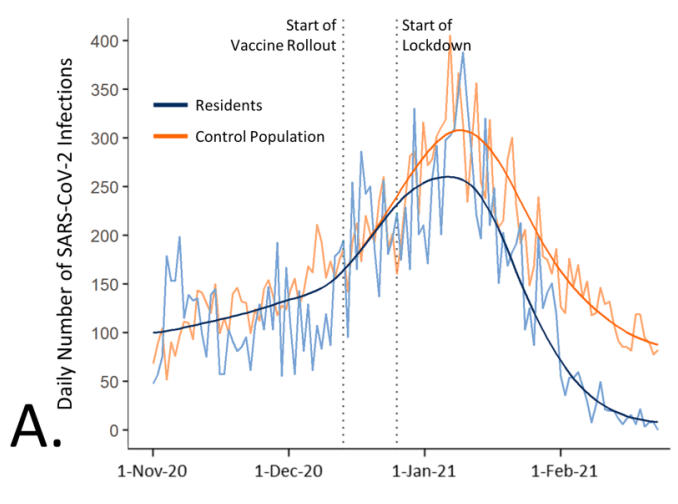

Health Care Workers

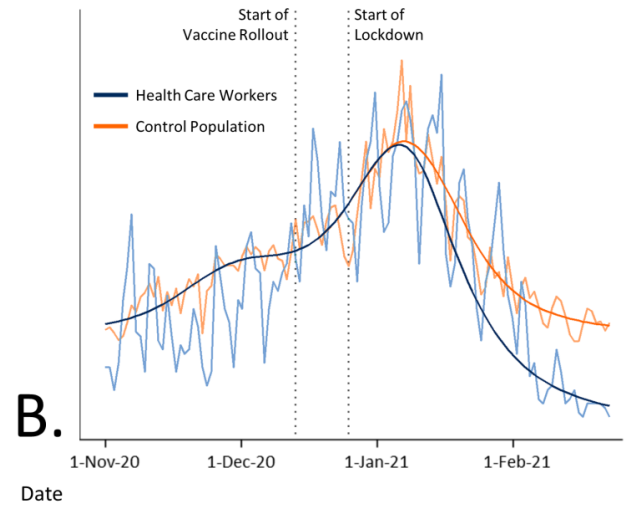

Figure 1. SARS-CoV-2 Infections Among Ontario LTC Residents and HCWs from November 1, 2020 to February 23, 2021

Line graphs of daily numbers and fitted curves of SARS-CoV-2 infections among Ontario LTC residents and HCWs from November 1, 2020 to February 23, 2021. Panel A shows daily numbers and fitted curves of SARS-CoV-2 infections in $L T C$ residents and a control population of unvaccinated community-dwelling older adults aged 70 years or older. Panel B shows daily numbers and fitted curves of SARS-CoV-2 infections in LTC HCWs and a control population of unvaccinated working age adults 18-64 years of age. Daily numbers of SARS-CoV-2 infections are standardized and expressed as a percentage relative to the number of SARS-CoV-2 infections observed on November 1, 2020. In the absence of vaccination, LTC residents would have still benefited from the overall decline in the incidence of community SARS-CoV-2 infections, realized with tightened public health restrictions. However, observed incidence dropped substantially more with vaccination. LTC, long-term care.

Table 1 shows that the vaccination of Ontario's LTC residents prevented an estimated 2,079 SARS-CoV-2 infections, 249 COVID-19 hospitalizations and 615 COVID-19 deaths. Vaccination of Ontario's LTC HCWs further prevented an estimated 590 SARS-CoV-2 infections, and 8 COVID-19 hospitalizations and one COVID-19 death between December 14, 2020, when the vaccine rollout in LTC homes started, and February 23, 2021.

\begin{tabular}{lccc}
\hline & Residents & Health Care Workers & Total \\
\hline Observed with Vaccine Rollout & & & \\
SARS-CoV-2 Infections & 5,350 & 2,320 & 7,670 \\
COVID-19 Hospitalizations & 642 & 30 & 672 \\
COVID-19 Deaths & 1,091 & 2 & 1,093 \\
Expected without Vaccine Rollout & & & \\
SARS-CoV-2 Infections & 7,429 & 2,910 & 10,339 \\
COVID-19 Hospitalizations & 891 & 38 & 929 \\
COVID-19 Deaths & 1,706 & 3 & 1,709 \\
Prevented by Vaccine Rollout & & & \\
SARS-CoV-2 Infections & 2,079 & 590 & 2,669 \\
COVID-19 Hospitalizations & 249 & 8 & 257 \\
COVID-19 Deaths & 615 & 1 & 616 \\
\hline
\end{tabular}

Table 1. Observed SARS-CoV-2 Infections, and COVID-19 Hospitalizations and Deaths and Prevented SARS-CoV-2 Infections, and COVID-19 Hospitalizations and Deaths Among Residents and HCWs of Ontario LTC homes from December 14, 2020 to February 23, 2021

Table presenting model-based estimates of observed, expected and prevented SARS-CoV-2 infections, and COVID-19 hospitalizations and deaths among residents and HCWs of Ontario LTC homes from November 1, 2020 to February 23, 2021. Observed SARS-CoV-2 infections were modelled based on the Public Health Case and Contact Management Solution (CCM), extracted on March 1, 2021. The estimated numbers of prevented events are based on a comparison of the estimated number of events in LTC residents and HCWs, compared to the number of events in statistical unvaccinated control populations. The estimated number of infections in the statistical unvaccinated control populations were modelled based on fitted slopes of the epidemic curves of SARS-CoV-2 infections in communitydwelling individuals aged $\geq 70$ years (for LTC residents), and the working age population aged 18-64 years (for LTC HCWs). LTC, long-term care.

Figure 2 shows the estimated impact of the vaccination rollout in LTC residents and HCWs on daily SARS-CoV-2 infections in Ontario LTC homes over time since the beginning of the COVID-19 vaccine rollout. Associated with an increase in vaccine coverage until February 13, 2021, when all LTC residents and staff were offered the 
first dose of a COVID-19 vaccine, differences between groups become more pronounced over time.

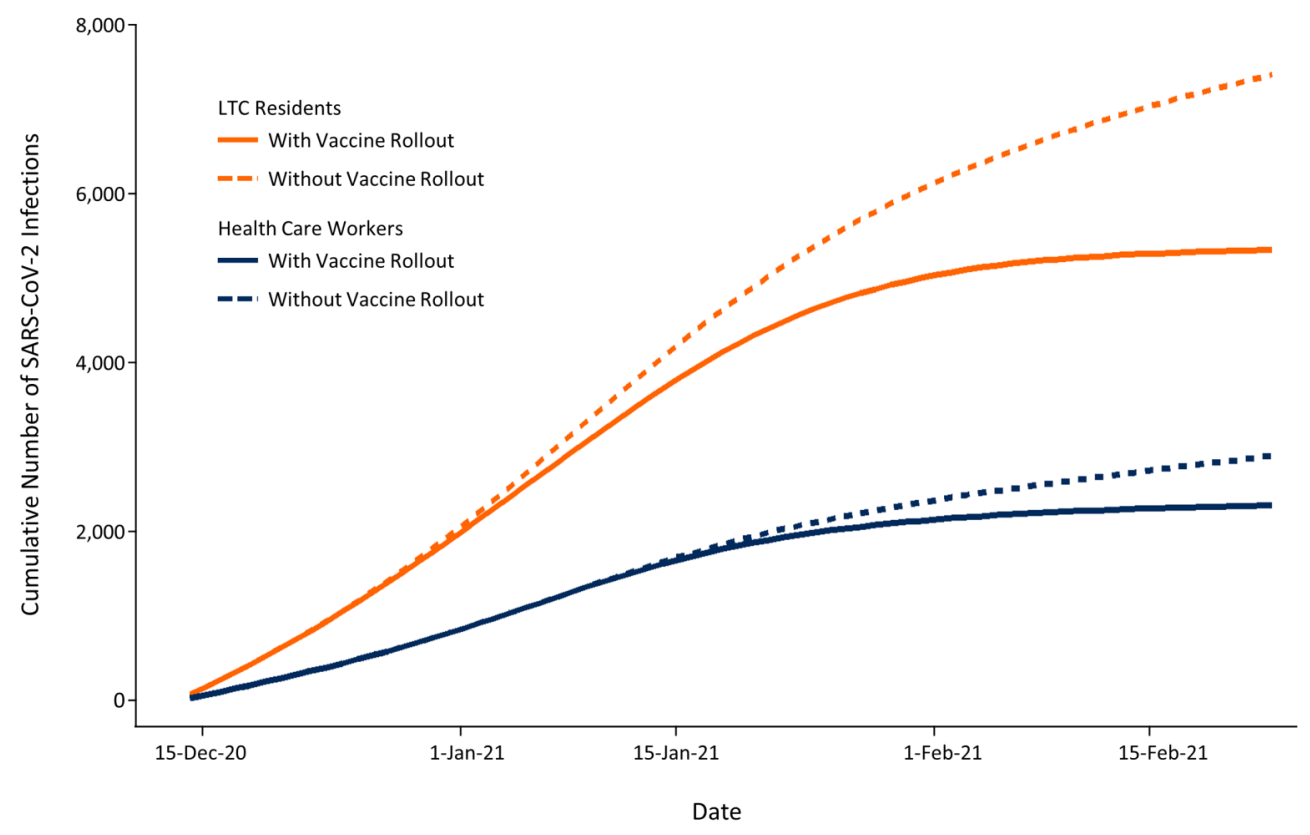

Figure 2. Cumulative SARS-CoV-2 Infections Among Ontario LTC Residents and HCWs from December 14, 2020 to February 23, 2021

Line graph showing the model-based cumulative numbers of observed SARS-CoV-2 infections during the vaccine rollout in LTC homes (solid lines) in comparison with the estimated SARS-CoV-2 infections that would have been expected in the absence of vaccination in statistical unvaccinated control populations (dashed lines) for LTC residents and HCWs. The estimated number of infections in statistical unvaccinated control populations were modelled based on fitted slopes of the epidemic curves of SARS-CoV-2 infections in community-dwelling individuals aged $\geq 70$ years (for LTC residents), and the working age population aged 18-64 years (for LTC HCWS). LTC, long-term care.

Figure 3 presents the estimated relative risk of SARS-CoV-2 infections and COVID-19 deaths in LTC residents and of SARS-CoV-2 infections in LTC HCWs. The effect of COVID-19 vaccines was substantial and became more pronounced with increasing time since the start of vaccine rollout in individual PHUs.

At 8 weeks from the start of vaccination, the estimated relative reduction in the risk of SARS-CoV-2 infection was $89 \%$ among LTC residents (relative risk $0.11,95 \% \mathrm{Cl}$ 0.07 to 0.15 ), and $79 \%$ in healthcare workers (relative risk $0.21,95 \% \mathrm{Cl} 0.15$ to 0.29 ). The estimated relative risk reduction of COVID-19 deaths was $96 \%$ among LTC residents (relative risk $0.04,95 \% \mathrm{Cl} 0.02$ to 0.08 ). These estimated reductions were above and beyond the estimated reductions associated with the province-wide lockdown enacted on December 26, 2020, and stay-at-home order implemented on January 14, 2021.

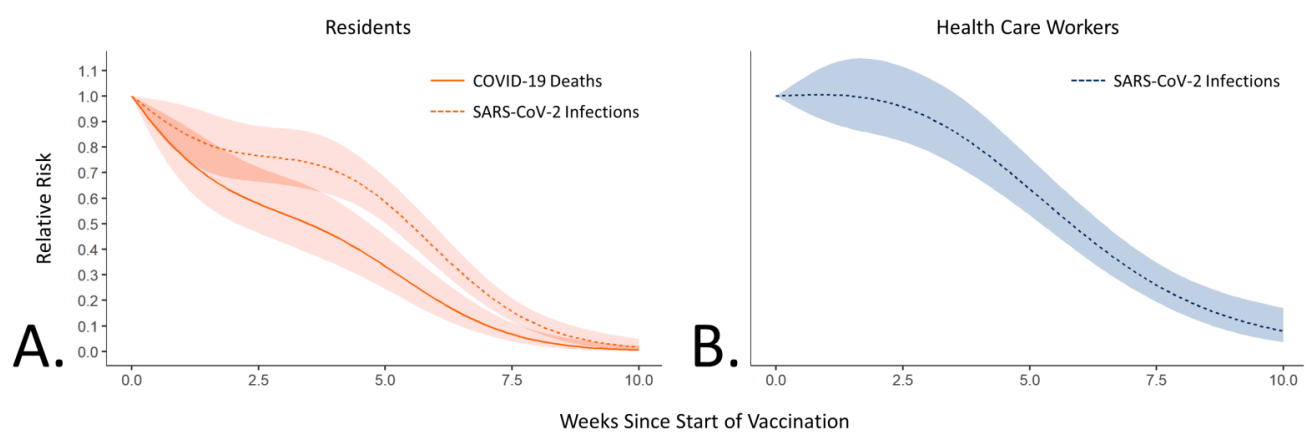

Figure 3. Relative Risk of SARS-CoV-2 Infections and COVID-19 Deaths in Ontario LTC residents and SARS-CoV-2 Infections in LTC HCWs as Compared with Appropriate Control Populations

Line graphs illustrating the relative risk of SARS-CoV-2 infections among LTC residents (A) and HCWs (B) as compared 
with statistical unvaccinated control populations. The estimated infections in statistical unvaccinated control populations were modelled based on fitted slopes of the epidemic curves of SARS-CoV-2 infections in LTC residents compared to community-dwelling individuals aged $\geq 70$ years, and SARS-CoV-2 infections in LTC HCWs compared to the Ontario working age population aged 18-64 years.

\section{Interpretation}

The rollout of COVID-19 vaccines in Ontario's LTC homes has substantially reduced SARS-CoV-2 infections, COVID-19 hospitalizations and deaths among LTC residents and HCWs. The estimated vaccine effects account for the reduction in community prevalence of SARS-CoV-2 infections following Ontario's province-wide lockdown enacted on December 26, 2020, and the stay-at-home order implemented on January 14, 2021.

This early real-world evidence demonstrates the effectiveness of COVID-19 vaccination among Ontario's LTC home population and workforce, with vaccination resulting in a relative reduction in SARS-CoV-2 incidence of 80 to $90 \%$ in both residents and HCWs compared to unvaccinated control populations.

Broad public health measures implemented in late December 2020 and early January 2021 acted synergistically with COVID-19 vaccination to prevent SARS-CoV-2 infections. This emphasizes that public health measures will need to be maintained alongside vaccination, until vaccine-based immunity has been afforded to the entire population.

These data highlight the importance of accelerating vaccine rollout to priority populations who are at disproportionately high risk of SARS-CoV-2 infection, COVID19 hospitalization and death. ${ }^{2,11}$ An earlier analysis projected that if all LTC residents received the first dose of a COVID-19 vaccine by January 31, 2021, this would have prevented a projected 600 COVID-19 cases and 115 deaths by March 31, 2021, as compared with providing at least one dose of a COVID-19 vaccine to all LTC residents by February 15, $2021 .^{2}$

While COVID-19 vaccine uptake has been more than $90 \%$ among Ontario LTC residents, the vaccine uptake among staff has been $68 \%$ as of March 5, 2021, which is lower than the reported vaccination intention rate of $80.4 \%$ among Ontario's unionized healthcare workers. ${ }^{12}$ Closing this gap is essential, and may require behavioural science informed education and communication about COVID-19 vaccines, ${ }^{13}$ as well as financial support such as paid time off, transportation for vaccination, and guaranteed paid sick leave in case of vaccine side effects that result in missed time from work. ${ }^{14}$

Emerging vaccine safety data from Ontario show that following the administration of 687,271 COVID-19 vaccine doses as of February 27, 2021, the most frequent adverse events reported to date were skin reactions, as well as pain and redness at the injection site. ${ }^{15}$ There were 12 serious adverse events reported, three of which were anaphylaxis. These safety data are consistent with early data from the United States suggesting the safety of mRNA vaccines, including for frail older adults in Ontario. $^{16,17}$

There are several ways in which the COVID-19 vaccine impact demonstrated in this analysis is different from the vaccine efficacy measured in randomized controlled vaccine trials. First, we studied a population of LTC residents, who were severely underrepresented in COVID-19 vaccine trials. Our findings therefore extend knowledge on the protective effect of vaccines in this population. Second, incomplete uptake of the vaccine in LTC residents and staff decreases the real-world impact. Third, a substantial number of Ontario LTC home residents may have already achieved some immunity to SARS-CoV-2 due to previous infection, thereby 
increasing the apparent benefit of the vaccine as compared with the statistical unvaccinated control population we derived. ${ }^{18}$ Fourth, our data are based on identified SARS-CoV-2 infections, and, as such, do not necessarily represent $100 \%$ of infections.

As seen in other Canadian and international jurisdictions, COVID-19 vaccination in Ontario has led to rapid declines in SARS-CoV-2 infections, COVID-19 hospitalizations and deaths among LTC residents and HCWs. ${ }^{19,20}$ Completing and maximizing the uptake of the full COVID-19 vaccine series according to recommended schedules will maximize the safety and well-being of Ontario's LTC residents and staff.

\section{Methods Used for This Science Brief}

\section{Study Population and Design}

We used a longitudinal study design to estimate the impact of COVID-19 vaccination on SARS-CoV-2 incidence, COVID-19 hospitalizations and deaths among Ontario LTC home residents and HCWs.

\section{Data Sources}

Data for this analysis were extracted from the Public Health Case and Contact Management Solution database for all PHUs on March 1, 2021. Only cases publicly reported between November 1, 2020 and February 23, 2021 were included. Information on the earliest start date and end date for administration of the first doses of COVID-19 vaccines for LTC residents and HCWs in each PHU was obtained from PHU websites, news articles, Twitter posts, and personal communications. This analysis includes all publicly announced vaccination completion dates up to and including Feb 23, 2021.

\section{Exposure}

The primary exposure was time since the start of COVID-19 vaccination within LTC homes in a PHU. Partial completion of PHU vaccination was interpolated from $0 \%$ completion on the day prior to vaccination initiation in the $\mathrm{PHU}, 50 \%$ completion on the midpoint date, to $100 \%$ on the date of completion itself.

\section{Control Populations}

A difference-in-differences analysis was performed by selecting control populations of individuals with a similar age group who had a very low probability of being vaccinated during the study period. The control population for LTC residents were individuals 70 years of age or older and living outside of LTC homes. The control population for LTC HCWs were individuals of working age between 18 and 64 years who were not coded as HCWs. Since HCWs outside of LTC homes may have also been vaccinated, these individuals were excluded from the comparisons.

\section{Outcomes}

The primary outcome was the daily number of LTC residents and HCWs with SARSCoV-2 infections in each PHU. The daily number of COVID-19 hospitalizations and deaths were secondary outcomes.

\section{Descriptive Statistics}

Summary statistics were used to describe LTC residents and HCWs by age, sex, and number of SARS-CoV-2 infections, COVID-19 hospitalizations and deaths. Counts and percentages were used to report categorical outcomes. 


\section{Estimation of Prevented SARS-CoV-2 Infections}

Two separate difference-in-differences analyses were developed, ${ }^{21}$ one for counts of LTC residents and their control population, and one for counts of LTC HCWs and their control population. The models were implemented using quasi-Poisson regression with each of the two models including 34 separate time-series panels corresponding to each PHU. Within each PHU, the baseline trend prior to COVID-19 vaccine rollout was parallel on the log-scale for the two groups, and the baseline trend was modeled using a penalized spline, ${ }^{22}$ with a knot for each two-week period. The effect of vaccination was implemented as a change in the growth rate following the start of LTC home vaccination. Daily numbers of SARS-CoV-2 infections were standardized and expressed as a percentage relative to the number of SARS-CoV-2 infections observed on November 1, 2020 (50 for LTC residents, 77 for communitydwelling individuals aged $\geq 70$ years, 22 for LTC HCWs, and 792 for the working age population aged $18-64$ years).

The change in growth rates was estimated as a penalized spline of the time since the start of COVID-19 vaccination, with knots for each 2-weeks of the post-intervention periods and was forced to intersect on the day of vaccination initiation. Based on these models, the estimated number of expected (without intervention), observed (given intervention), and prevented cases of SARS-CoV-2 infection was determined by comparing the predicted incidence in the absence of vaccination, versus the observed incidence given the COVID-19 vaccination start and completion date for every PHU.

\section{Estimation of Prevented COVID-19 Hospitalizations and Deaths}

To estimate the impact of vaccination on COVID-19 deaths among LTC residents, an additional difference-in-differences model was used, using case-fatality as the outcome. The case fatality model had a single spline for date (not PHU specific), and a similar spline for time since start of vaccination. The estimated number of deaths was the product of the estimated difference-in-differences of infection, multiplied by the difference-in-differences of case fatality. The cumulative number of deaths prevented was then calculated in the same way as infections above.

The estimated case hospitalization rate (CHR) for LTC residents and LTC healthcare workers, and the estimated case fatality rate (CFR) for LTC healthcare workers were calculated from CCM plus data from November 1, 2020 to December 31, 2020, as the observed proportions (CHR for residents, 0.12; CHR for LTC HCWs, 0.01; CFR for LTC HCWs, 0.001). The estimated number of COVID-19 hospitalizations and deaths prevented among LTC HCWs was calculated by multiplying the estimated number of cases prevented with the case fatality rate and hospitalization rate, respectively.

\section{Author Contributions}

$K A B, N M S, P J$ and PAR conceived the Science Brief and wrote the first draft. KAB and TV performed the analysis. All authors revised the Science Brief critically for important intellectual content, and approved the final version.

\section{References}

1. Stall NM, Brown KA, Maltsev A, et al. COVID-19 and Ontario's long-term care homes (full brief). Sci Briefs Ont COVID-19 Sci Advis Table. 2021;1(7). https:// doi.org/10.47326/ocsat.2021.02.07.1.0

2. Stall NM, McGeer A, Maltsev A, et al. The Impact of the Speed of Vaccine Rollout on COVID-19 Cases and Deaths in Ontario Long-Term Care Homes. Ontario COVID-19 
Science Advisory Table; 2021. https://doi.org/10.47326/ocsat.2021.02.08.1.0

3. Ontario Long-Term Care Association. The role of long-term care. oltca.com. Published 2020. Accessed December 8, 2020. https://www.oltca.com/oltca/OLTCA/ Public/LongTermCare/FactsFigures.aspx

4. Government of Canada SC. Data tables, 2016 census. Type of collective dwelling (16), age (20) and sex (3) for the population in collective dwellings of Canada, provinces and territories, 2016 Census - 100\% Data.

5. Long-Term Care Staffing Study Advisory Group. Long-Term Care Staffing Study. Ministry of Long-Term Care; 2020:51. https://files.ontario.ca/mltc-long-term-carestaffing-study-en-2020-07-31.pdf

6. Getting a COVID-19 vaccine in Ontario. COVID-19 in Ontario. https://covid19.ontario.ca/getting-covid-19-vaccine-ontario

7. Ontario declares second provincial emergency to address COVID-19 crisis and save lives. Ontario Newsroom. Published January 12, 2021. Accessed February 19, 2021. https://news.ontario.ca/en/release/59922/ontario-declares-second-provincial -emergency-to-address-covid-19-crisis-and-save-lives

8. Government of Ontario. COVID-19 response framework: keeping Ontario safe and open. Ontario.ca. Published November 3, 2020. Accessed December 6, 2020. https://www.ontario.ca/page/covid-19-response-framework-keeping-ontario-safeand-open

9. Government of Ontario. COVID-19 vaccination update. Technical Briefing presented at the: January 13, 2021; Ontario. Accessed January 19, 2020. https:// files.ontario.ca/moh-covid-19-vaccine-technical-briefing-en-january-13-2021-202101-13.pdf

10. Government of Ontario. COVID-19 vaccines for Ontario. COVID-19 (coronavirus) in Ontario. https://covid-19.ontario.ca/covid-19-vaccines-ontario

11. Brown KA, Stall NM, Joh E, et al. A strategy for the mass distribution of COVID-19 vaccines in Ontario based on age and neighbourhood. Sci Briefs Ont COVID-19 Sci Advis Table. 2021;2(10). https://doi.org/10.47326/ocsat.2021.02.10.1.0

12. Desveaux L, Savage RD, Tadrous M, et al. Beliefs associated with intentions of non-physician healthcare workers to receive the COVID-19 vaccine in Ontario, Canada. medRxiv. Published online February 26, 2021:2021.02.19.21251936. https://doi.org/10.1101/2021.02.19.21251936

13. Presseau J, Desveaux L, Allen U, et al. Behavioural science principles for supporting COVID-19 vaccine confidence and uptake among Ontario health care workers. Sci Briefs Ont COVID-19 Sci Advis Table. 2021;2(12). https:// doi.org/10.47326/ocsat.2021.02.12.1.0

14. Buttenheim AM. SARS-CoV-2 vaccine acceptance: we may need to choose our battles. Ann Intern Med. 2020;173(12):1018-1019. https://doi.org/10.7326/M206206

15. Public Health Ontario. Adverse events following immunization (AEFIs) for COVID19 in Ontario: December 13, 2020 to February 27, 2021. Public Health Ontario. https://www.publichealthontario.ca/-/media/documents/ncov/epi/covid-19-aefireport.pdf?la=en

16. Shimabukuro TT, Cole M, Su JR. Reports of anaphylaxis after receipt of mRNA COVID-19 vaccines in the US-December 14, 2020-January 18, 2021. JAMA. Published online February 12, 2021. https://doi.org/10.1001/jama.2021.1967 
17. Blumenthal KG, Freeman EE, Saff RR, et al. Delayed Large Local Reactions to mRNA-1273 Vaccine against SARS-CoV-2. N Engl J Med. Published online March 3, 2021. https://doi.org/10.1056/NEJMc2102131

18. Ministry of Health / Long-Term Care. LTC Analysis. Published February 2021. http://www.Itccommission-commissionsld.ca/presentations/pdf/

Government_of_Ontario_Michael_Hillmer_February_19_2021.pdf

19. Government of Quebec. Preliminary Data on Vaccine Effectiveness and Supplementary Opinion on the Strategy for Vaccination Against COVID-19 in Quebec in a Context of Shortage. inspq.qc.ca. Published February 12, 2021. https:// www.inspq.qc.ca/en/publications/3111-vaccine-effectiveness-strategy-vaccinationshortage-covid19

20. Rossman H, Shilo S, Meir T, Gorfine M, Shalit U, Segal E. Patterns of COVID-19 pandemic dynamics following deployment of a broad national immunization program. medRxiv. Published online February 9, 2021:2021.02.08.21251325. https://doi.org/10.1101/2021.02.08.21251325

21. Dimick JB, Ryan AM. Methods for evaluating changes in health care policy: the difference-in-differences approach. JAMA. 2014;312(22):2401-2402. https:// doi.org/10.1001/jama.2014.16153

22. Pedersen EJ, Miller DL, Simpson GL, Ross N. Hierarchical generalized additive models in ecology: an introduction with mgcv. PeerJ. 2019;7:e6876. https:// doi.org/10.7717/peerj.6876 